\title{
Hemicorporectomía y equipamiento protésico. Reporte de un caso a los 15 años de seguimiento
}

\author{
Walter Cerrutti," Gerardo L. Gallucci," José D. Freire" + \\ "Centro de Ortopedia y Traumatología Quilmes, Buenos Aires, Argentina \\ ${ }^{* * *}$ Servicio de Ortopedia y Traumatología, Hospital Italiano de Buenos Aires, Ciudad Autónoma de Buenos Aires, Argentina \\ ${ }^{\dagger}$ Fallecido
}

\begin{abstract}
RESUMEN
La hemicorporectomía o amputación translumbar es una cirugía radical indicada en casos de tumores, osteomielitis crónicas y traumas graves de la pelvis, y también, en pacientes parapléjicos con úlceras por decúbito intratables. La tasa de mortalidad por este procedimiento es del $50 \%$ y la gran mayoría de los pacientes que sobreviven quedan confinados a una silla de ruedas. El equipamiento de todo el hemicuerpo inferior es sumamente complejo. Comunicamos el caso de un paciente con una hemicorporectomía, secuela de una osteomielitis pélvica, su equipamiento completo posterior y su evolución a los 15 años de seguimiento. Palabras clave: Hemicorporectomía; amputación translumbar; prótesis; osteomielitis pelviana.
\end{abstract}

Nivel de Evidencia: IV

\section{Hemicorporectomy and Prosthetic Equipment. Report of a Case at 15 Years of Follow-up}

\begin{abstract}
Hemicorporectomy or translumbar amputation is a radical surgery indicated in localized pelvic tumors, chronic osteomyelitis, severe trauma, and intractable decubitus ulcers in paraplegic patients. It has a death rate of about $50 \%$ and most of the patients are confined to a wheelchair. The equipment of the entire lower body is extremely complex. We report a case of a patient with a hemicorporectomy, sequel to pelvic osteomyelitis, his subsequent complete equipment, and his evolution after 15 years of follow-up.

Key words: Hemicorporectomy; translumbar amputation; prosthesis; pelvic osteomyelitis.
\end{abstract}

Level of Evidence: IV

\section{INTRODUCCIÓN}

La hemicorporectomía o amputación translumbar incluye la amputación de la pelvis y las extremidades inferiores a través de la columna lumbar con la concomitante transección de la aorta, la vena cava inferior y la médula espinal. Se acompaña, además, de la correspondiente derivación urinaria e intestinal.

Fue propuesta originalmente por Kredel, en $1950,{ }^{1}$ y Kennedy y cols. la llevaron a cabo, con éxito, por primera vez, en $1960 .^{2}$

Al principio, la hemicorporectomía se propuso para tumores graves invasivos de la pelvis, aunque también se ha indicado para casos de trauma grave de la pelvis y las extremidades inferiores, ${ }^{3}$ malformaciones vasculares, ${ }^{4}$ oclusión aórtica aguda, ${ }^{5}$ fístulas perianales y escrotales recurrentes, ${ }^{6}$ y osteomielitis pélvica terminal. ${ }^{7,8}$

La hemicorporectomía es una cirugía de altísimo riesgo, la tasa de mortalidad perioperatoria supera el 50\%. ${ }^{9}$ Este riesgo obedece a lo extensa que es la cirugía, la pérdida de sangre y las fallas cardiovasculares después de la separación del torso de la pelvis. Hasta la fecha, se han publicado 66 hemicorporectomías. ${ }^{10}$ La indicación más frecuente ha sido una causa tumoral y, la menos frecuente, sepsis u osteomielitis pélvicas terminales. 
La mayoría de los pacientes con hemicorporectomía que logran sobrevivir son confinados a una silla de ruedas con una inclusión parcial en las actividades de la vida diaria. El equipamiento de los miembros inferiores en estos pacientes es muy complejo y, en general, poco tolerado.

El objetivo de este artículo es comunicar el caso de un paciente con hemicorporectomía, secuela de una osteomielitis pélvica, su equipamiento posterior y su evolución a los 15 años de seguimiento.

\section{CASO CLÍNICO}

Hombre de 25 años que había sufrido un accidente de moto cinco años atrás. La lesión le produjo una fractura a nivel de D10 y un cuadro de paraplejia con nivel sensitivo compatible con la lesión. Su estado neurológico se clasificó como Frankel A. Los malos cuidados higiénicos le provocaron escaras múltiples en piernas, sacro y trocánteres, que obligaron a realizar varios procedimientos quirúrgicos, inclusive la amputación infrarrotuliana de ambas piernas (Figura 1). Este cuadro irreversible continuó evolucionando y finalizó con el desarrollo de una osteomielitis pélvica masiva que requirió la colocación de una talla vesical permanente y una cirugía de Hartman (colostomía).

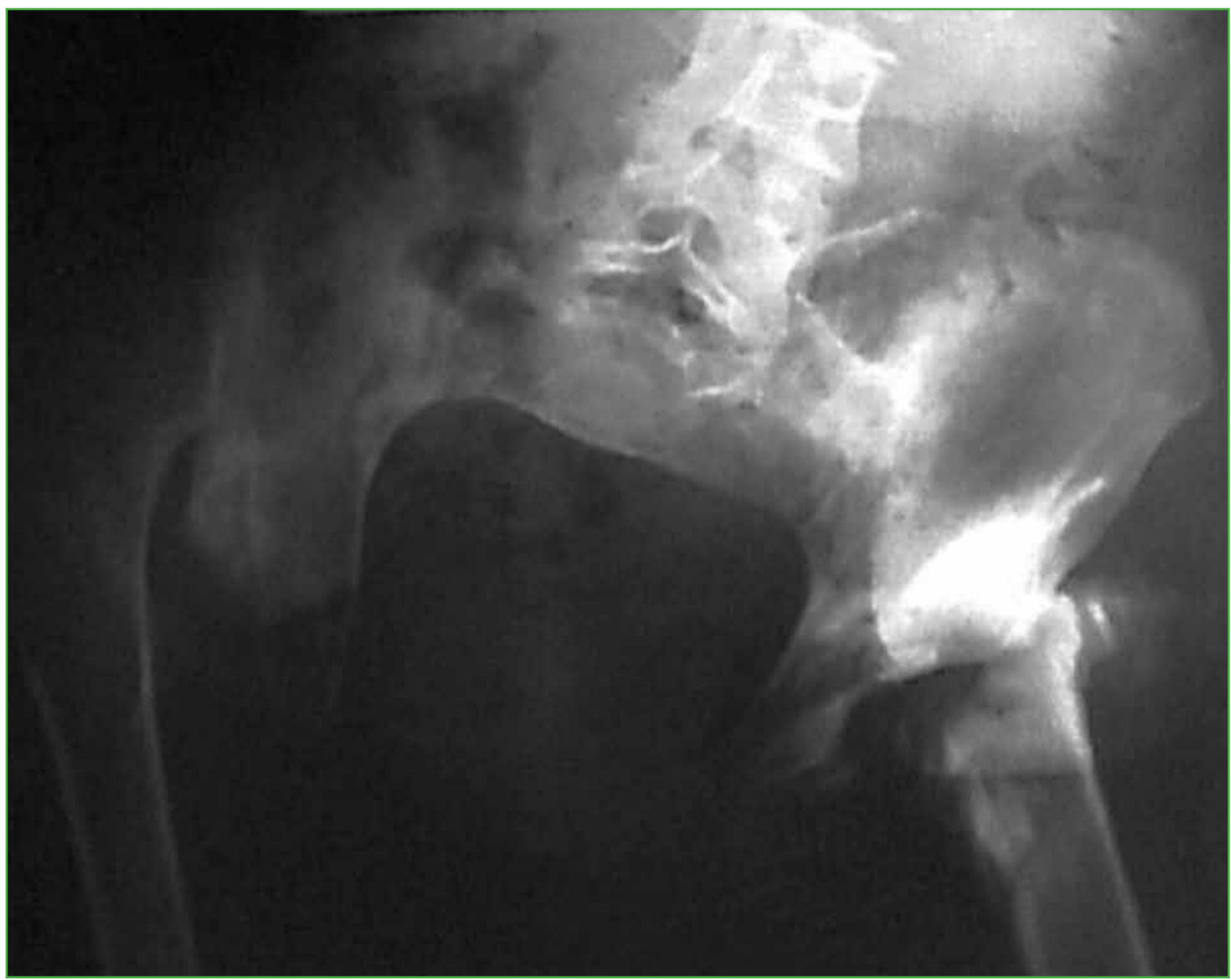

Figura 1. Radiografía de pelvis de frente que muestra la osteomielitis.

Durante los últimos años, la secreción purulenta por su talla vesical era permanente y tuvo numerosas internaciones para recibir tratamientos antibióticos, realizar recambios de talla vesical y drenajes de empiemas pélvicos. Desarrolló, además, una úlcera sacra de gran tamaño. El estado nutricional preoperatorio, según la clasificación de Gómez, ${ }^{11}$ era del 58\%, que corresponde a una desnutrición severa, con un índice de masa corporal de 16. Su calidad de vida se determinó con el Short Form-36 Health Survey, ${ }^{12}$ que evalúa la discapacidad de 0 (máxima discapacidad) a 100 (mínima discapacidad). El resultado obtenido fue de 15 puntos. 
En esta situación terminal, consulta en nuestro Servicio y la posibilidad de realizar una hemicorporectomía surgió como una alternativa terapéutica válida. Se solicitó una interconsulta psicológica y se le explicó al paciente el procedimiento detalladamente. Después de obtener la aceptación y el consentimiento informado se procedió a su internación.

El procedimiento estuvo a cargo de un equipo multidisciplinario (traumatología, cirugía general y urología) encabezado por uno de los autores (JF), en un solo tiempo quirúrgico bajo anestesia general (Figuras 2 y 3 ).

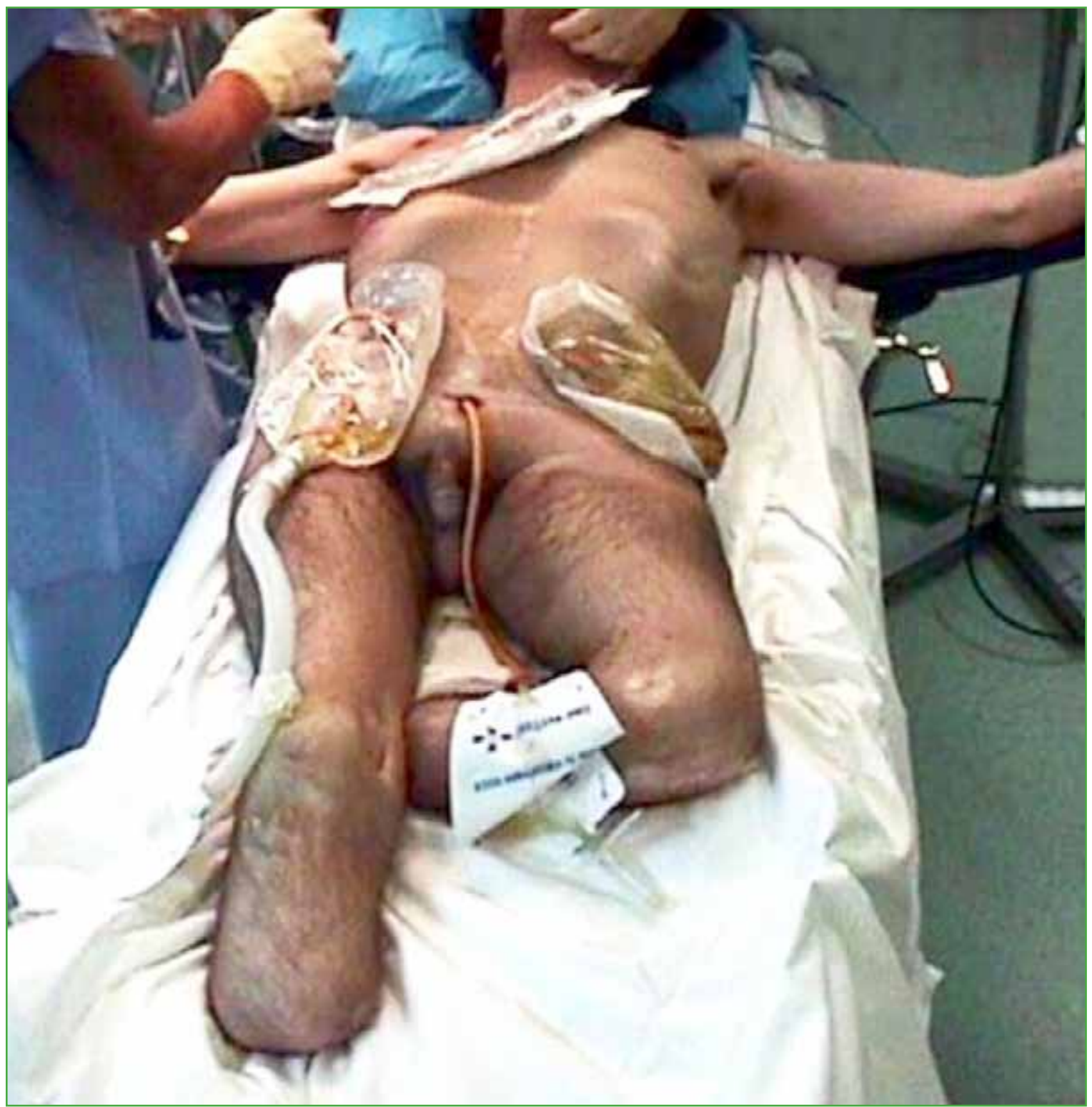

Figura 2. Estado del paciente en el momento de la cirugía. 


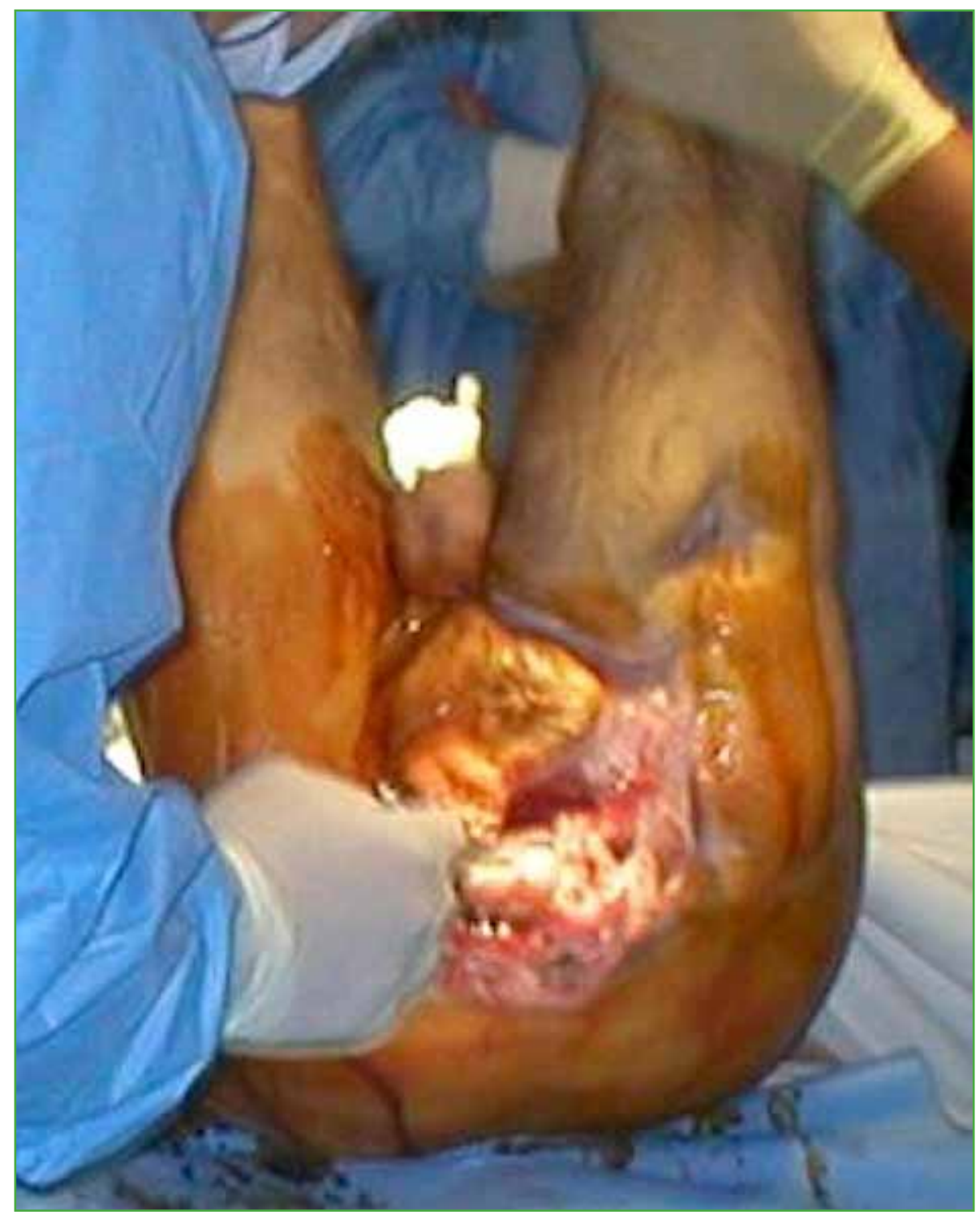

Figura 3. Escara sacra.

La cirugía comenzó con el paciente en decúbito dorsal y un abordaje ilioinguinal bilateral ampliado a proximal con la finalidad de crear un colgajo anterior que luego cubra el defecto posterior (Figura 4). Incluyó una colostomía y una ureteroileostomía. Se procedió a la ligadura de la vena cava y de ambas arterias ilíacas comunes. Se disecó y ligó el plexo de Batson y se realizó la desarticulación en L3-L4. Se administró lidocaína al 1\% intraneural para evitar el shock neurológico y se efectuó la resección de las raíces con electrobisturí bipolar. La duramadre se cerró con Prolene 6-0 y dicho cierre se confirmó mediante maniobras de Valsalva. 


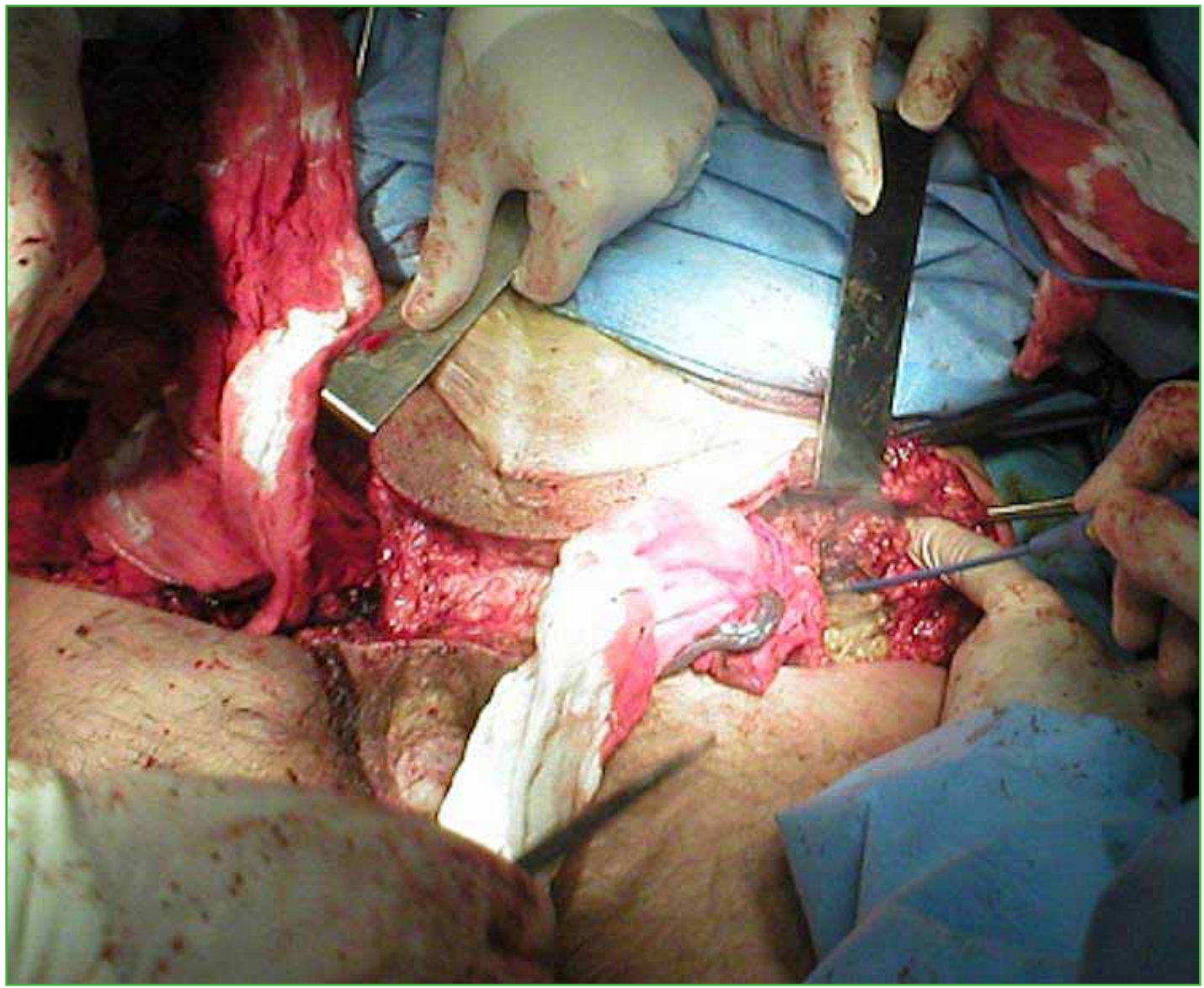

Figura 4. Abordaje ilioinguinal bilateral.

Luego se colocó al paciente en decúbito ventral y se procedió al cierre del colgajo posterior (Figura 5).

La cirugía se prolongó 11 h y fue necesario administrar 11 unidades de glóbulos rojos.

El paciente fue llevado a la Unidad de Cuidados Intensivos donde permaneció cinco días, tras lo cual fue trasladado a una sala de internación general en la que estuvo seis días. Se le administraron antibióticos por vía intravenosa: vancomicina $1 \mathrm{~g}$ cada $12 \mathrm{~h}$ e imipenem $500 \mathrm{mg}$ cada $12 \mathrm{~h}$. Luego mantuvo un plan antibiótico por seis semanas con amoxicilina/ácido clavulánico $250 \mathrm{mg}$ y $500 \mathrm{mg}$, respectivamente, cada $12 \mathrm{~h}$.

Seis días después de la cirugía, tuvo una dehiscencia de la herida que requirió múltiples curaciones.

Su cuadro séptico inicial mejoró notablemente y, a los cinco meses de la operación, se le realizó una neovejiga con técnica de Bricker y, en el mismo acto quirúrgico, se talló un colgajo local para la cobertura del defecto residual. 


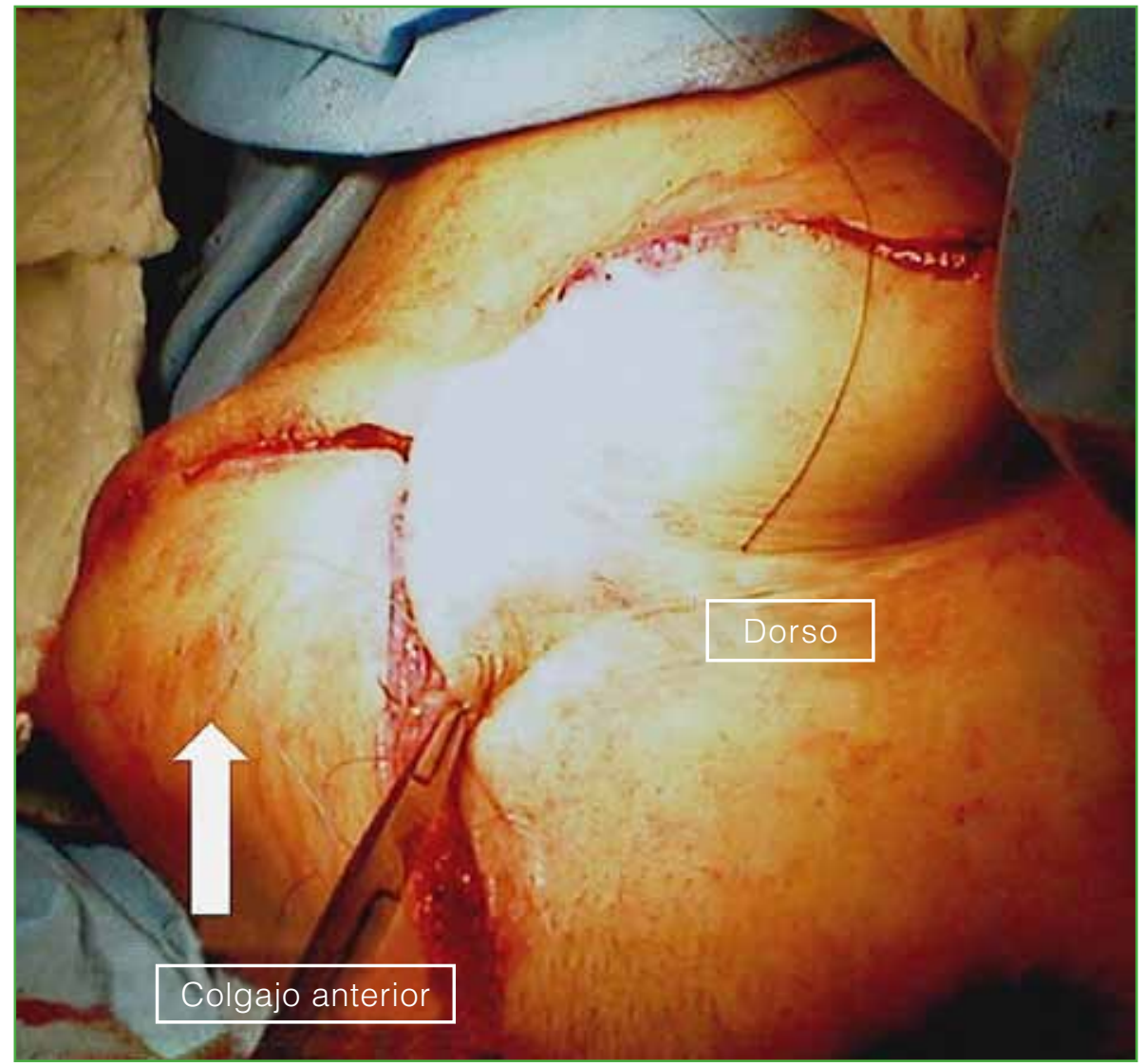

Figura 5. Colgajo para cobertura posterior.

\section{Equipamiento}

Durante los primeros meses y mientras se esperaba la cicatrización de la herida, se comenzó con un plan de fortalecimiento de los miembros superiores asistido por terapistas. Una vez lograda la cicatrización completa de la herida, se pasó a la etapa de equipamiento preprotésico. En esta etapa, se confeccionó una cesta de yeso con apoyo costal recubierta en su interior con Polyform. Luego, se le fijó una base plana de madera cubierta con yeso, que le permitía al paciente permanecer en posición vertical por intervalos de una hora. Posteriormente, se retiraba la cesta, se evaluaban las zonas de presión costal y se realizaban las correcciones necesarias. El uso incrementado progresivo de la cesta continuó durante los dos meses siguientes, hasta que el paciente logró un uso permanente de $6 \mathrm{~h}$, con buena tolerancia (Figura 6).

Luego del tercer mes de uso, se confeccionó un corsé cesta de un laminado de acrílico y carbono, con acolchado interior de Polyform, con apoyo costal y un método de suspensión mediante tiradores con hebilla con velcro (Figura 7). 


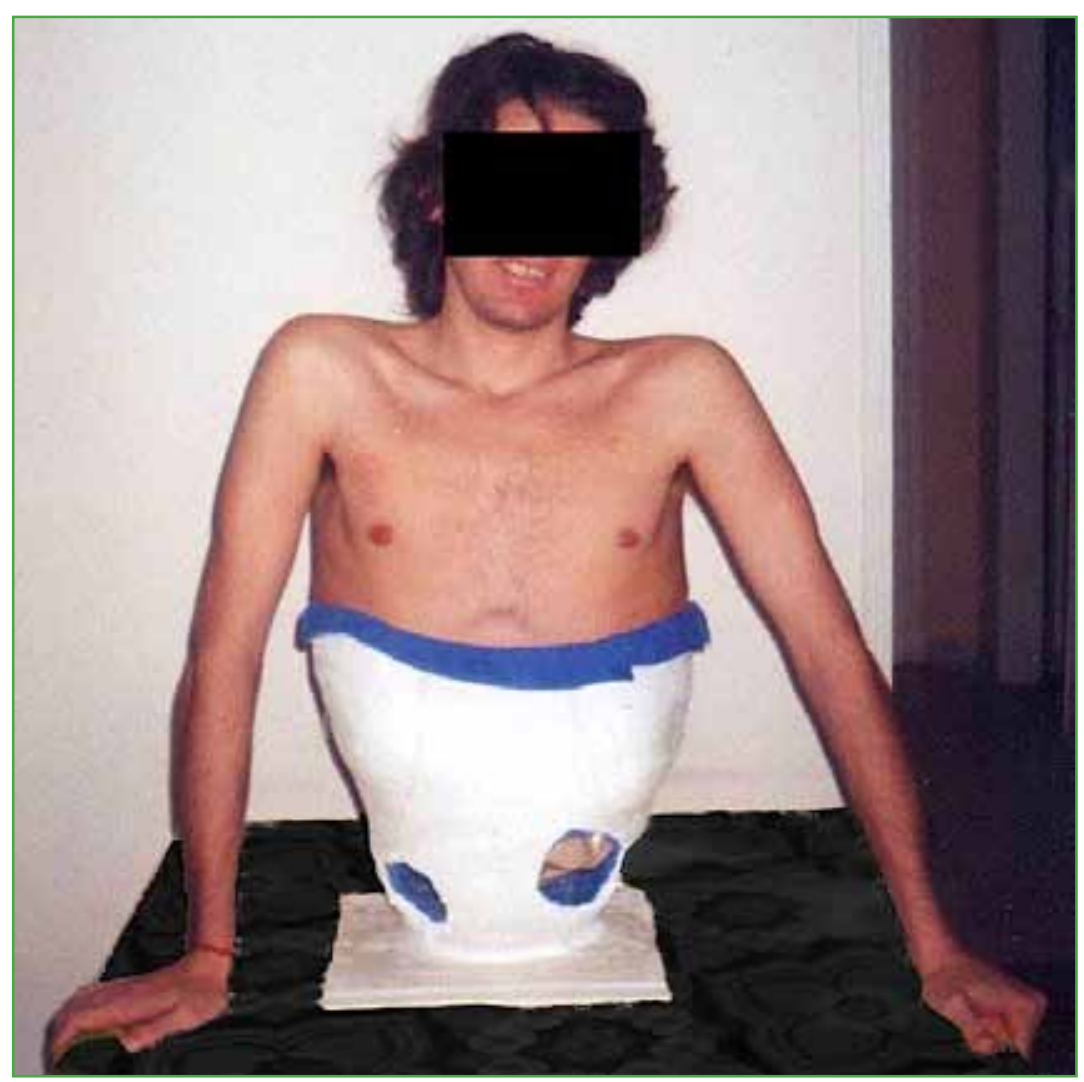

Figura 6. Confección de la cesta de yeso con apoyo plano.

Figura 7. Confección del corsé cesta de un laminado de acrílico y carbono, con acolchado interior de Polyform y apoyo costal.

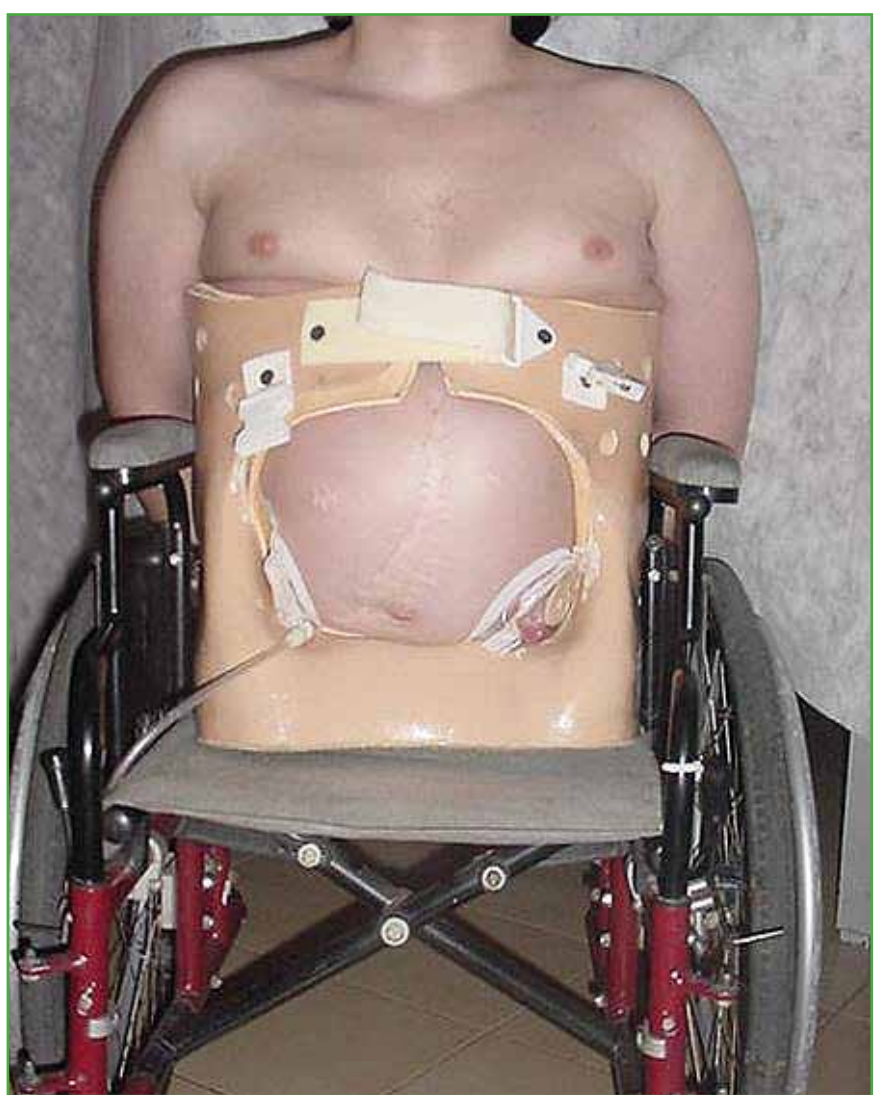


Después de esta etapa, se adosó al corsé el resto de las prótesis. Se tomaron las medidas antropométricas para devolver su talla original. Para las articulaciones de las caderas se utilizó una prótesis 7E5 de Ottobock (Ottobock SE \& Co. KGaA, Alemania) con un sistema de trabas manuales que le permitía bloquear la cadera en extensión para caminar y desbloquearla para sentarse. Para las articulaciones de las rodillas se utilizó una prótesis policéntrica mecánica 3R20 de Ottobock (Ottobock SE \& Co. KGaA, Alemania) con módulos de unión en titanio y, para los pies, una prótesis dinámica SACH+ Ottobock (Ottobock SE \& Co. KGaA, Alemania) con núcleo de madera (Figura 8).

Una vez montada la prótesis se inició el proceso de aprendizaje para su utilización. Lograr la bipedestación fue el objetivo inicial del tratamiento, luego, la deambulación (de manera pendulante con andador), y finalmente sentarse y subir y bajar escalones. Todo este proceso duró aproximadamente seis meses hasta que el paciente logró su independencia. Posteriormente, se logró que pudiera conducir vehículos adaptados. Por último, la prótesis fue recubierta con una espuma plástica para mejorar su estética (Figura 9).

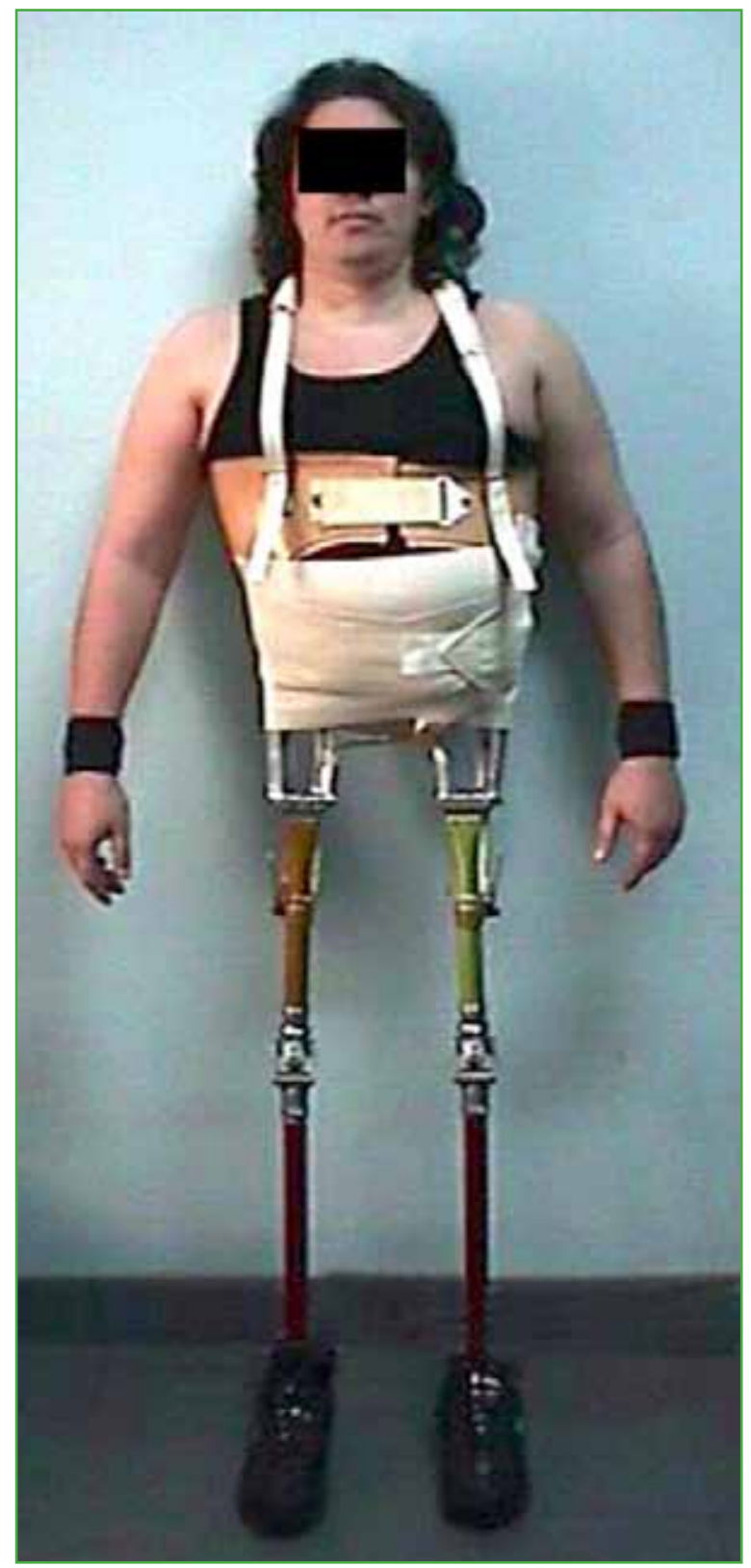

Figura 8. Paciente con equipamiento completo.

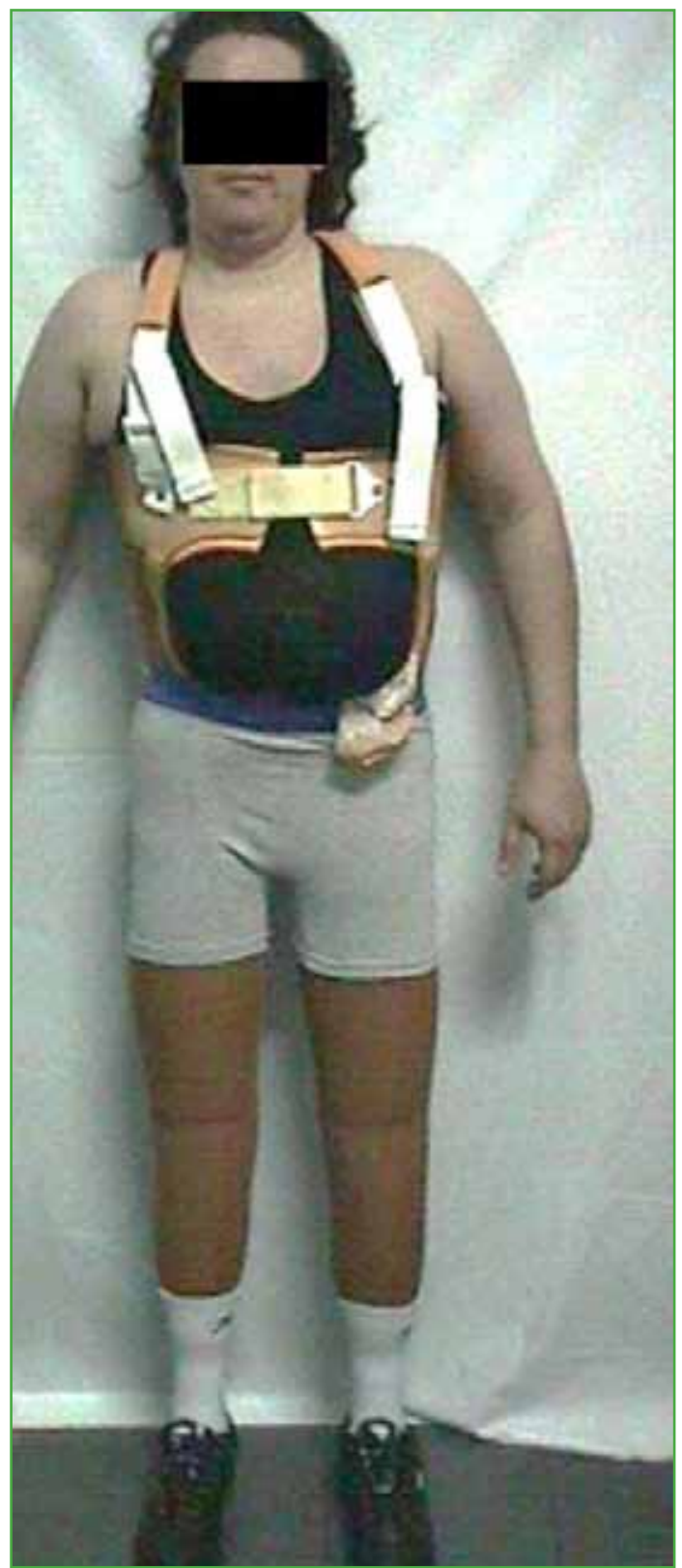

Figura 9. Aspecto estético del implante. 


\section{RESULTADOS}

A los 15 años de la cirugía, se citó al paciente para un control. Se realizó una evaluación funcional genérica con el Índice de Barthel ${ }^{13}$ y una específica con el Locomotor Capabilities Index. ${ }^{14}$

El Índice de Barthel, también conocido como Índice de Discapacidad de Maryland, valora el nivel de independencia del paciente en algunas actividades básicas de la vida diaria. La interpretación sugerida por Shah y cols. ${ }^{15}$ sobre el puntaje del Índice de Barthel es: 0-20: dependencia total, 21-60: dependencia severa, 61-90: dependencia moderada, 91-99: dependencia escasa y 100: independencia. El puntaje de nuestro paciente fue 80 (dependencia moderada).

El Locomotor Capabilities Index es una evaluación compuesta de 14 preguntas que determina la capacidad del paciente de realizar determinadas actividades con el uso de una prótesis. La variación del puntaje es de 0 (menor puntaje) a 56 (mayor puntaje). El paciente obtuvo un puntaje de 31. El Short Form-36 Health Survey arrojó 74 puntos.

Por indicación médica de control, se le solicitó un ecocardiograma para evaluar la función cardíaca a los 14 años de seguimiento. El volumen de eyección era de $70 \mathrm{mmHg}$ con una frecuencia cardíaca de 60 lat./minuto. En ese momento, se lo sometió también a una prueba de esfuerzo caminando $100 \mathrm{~m}$ con el andador. La frecuencia cardíaca se elevó a 96 lat./min, lo que representa un aumento del gasto cardíaco del $60 \%$ con la deambulación.

Desde el punto de vista social, el paciente logró una reincorporación total. La marcha que ha logrado es de tipo pendular con la ayuda del andador. Vive en pareja, trabaja en un taller, maneja un automóvil adaptado, realiza actividad física diaria y es corredor de autos (Figura 10).

Refiere estar sumamente agradecido y conforme con el procedimiento realizado y no duda en aconsejarlo a pacientes que estén en su misma situación terminal.

\section{DISCUSIÓN}

Aunque originalmente la hemicorporectomía se propuso para el tratamiento de tumores invasivos de la pelvis, la mejora en la cirugía oncológica y los avances de la quimioterapia y radioterapia han hecho que esa indicación sea, en la actualidad, menos frecuente. La mayoría de los últimos estudios publicados incluyen pacientes con una patología traumática grave, grandes úlceras crónicas por decúbito u osteomielitis pélvica que no responden a ningún tipo de tratamiento. . $16,17^{2}$

Para lograr resultados exitosos con baja morbilidad y mortalidad, es esencial utilizar un enfoque multidisciplinario. Esto implica la coordinación de varios servicios quirúrgicos, como cirugía general, ortopedia, cirugía plástica, neurocirugía, urología y rehabilitación.

Es fundamental, además, el manejo clínico perioperatorio, especialmente el conocimiento de los cambios en el equilibrio ácido-base, la función cardiovascular y la función respiratoria.

Las comorbilidades asociadas con la hemicorporectomía pueden incluir: íleo paralítico, obstrucción intestinal, úlceras gastroduodenales por estrés, infecciones urinarias, meningitis, trastornos metabólicos y dehiscencias de las heridas. ${ }^{7,818}$ En los primeros informes de hemicorporectomía, la muerte después de la cirugía se atribuía, en gran medida, al tumor, además de la gran pérdida de superficie corporal. Los efectos de la disipación del calor y de la regulación de la temperatura hacen que estos pacientes tengan una capacidad disminuida para regular la temperatura corporal, especialmente durante la actividad física. La ligadura de los vasos femorales comunes y de los nervios causa un aumento agudo de la resistencia vascular sistémica y la poscarga cardíaca, lo que puede precipitar la insuficiencia cardíaca y el edema pulmonar.

Finalmente, el cuidado meticuloso de las heridas, la prevención de las úlceras por presión, la rehabilitación extensa y el apoyo psicológico y emocional son críticos en el período posoperatorio.

La alta tasa de complicaciones también se debe al mal estado nutricional de estos pacientes. Resulta muy complejo intentar mejorar la nutrición en el preoperatorio de pacientes con osteomielitis pélvicas terminales, heridas crónicamente exudativas y además grandes desequilibrios del medio interno, a pesar de la suplementación y el tratamiento nutricional agresivo por parte de los nutricionistas.

Janis y cols. ${ }^{16}$ publicaron su experiencia con 11 pacientes sometidos a hemicorporectomía por secuelas de osteomielitis pélvica y comunicaron una supervivencia del 53\% al final del seguimiento.

En una revisión de 14 hemicorporectomías por osteomielitis intratables, Barnett y cols. ${ }^{19}$ informaron solo dos muertes al final del seguimiento, pero una extensa estancia hospitalaria (un promedio de 127 días), con una pérdida de sangre que variaba entre 2 y 12 litros. 

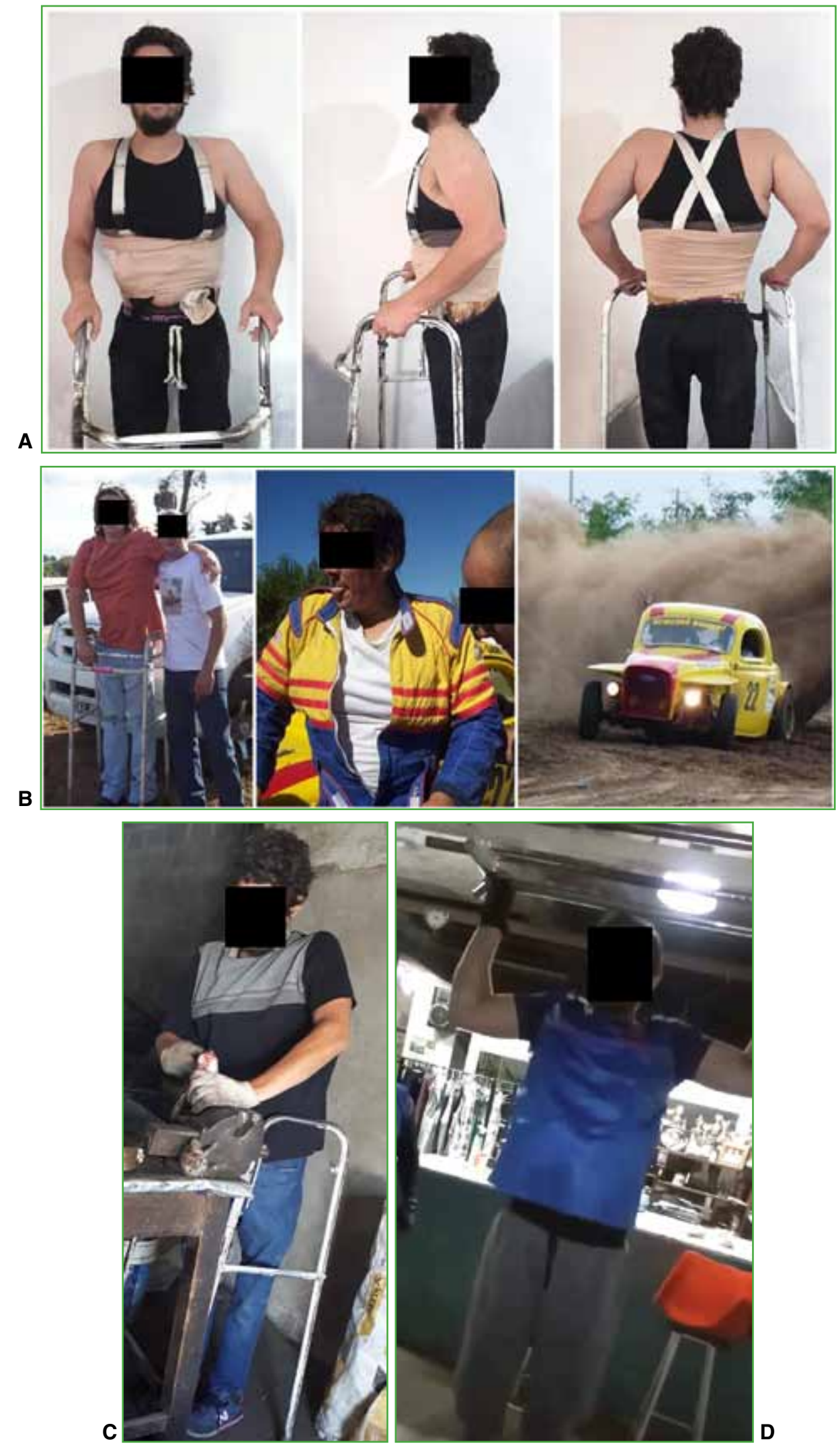

Figura 10. A. Estado actual del paciente con un andador. B. En competencias automovilísticas. C. Trabajando en su taller. D. En el gimnasio. 
Nuestro paciente tuvo complicaciones en la herida posiblemente relacionadas con la infección y su mal estado nutricional. En cuanto mejoró la cicatrización de la herida evolucionó favorablemente.

El objetivo de la rehabilitación después de la hemicorporectomía es restaurar la independencia y la movilidad del paciente. Pero esto no es sencillo de lograr, ya que aproximadamente el $40 \%$ finalmente no acepta la prótesis.

La mayoría de los pacientes descritos en la bibliografía son equipados con una cesta de apoyo costal y logran movilizarse en silla de ruedas. ${ }^{16,20}$ El equipamiento de los miembros inferiores está asociado a muchas complicaciones. La baja velocidad de la marcha permitida por la prótesis y la necesidad de ayuda con un andador son frecuentes motivos para el rechazo. Se han reconocido que las secuelas fisiológicas y psicológicas complejas de este procedimiento también complican el manejo protésico de este grupo de pacientes.

Una prótesis debe cumplir con ciertas necesidades de rehabilitación funcional y psicosocial y ajustarse a determinados criterios: permitir una postura erguida y estable con máxima libertad de la extremidad superior, mantener el soporte del cuerpo sin ejercer una presión intrabdominal excesiva y permitir la libre respiración y el acceso a los drenajes urológicos e intestinales sin obstáculos. La confección de una prótesis total de la parte inferior del cuerpo plantea grandes desafíos. ${ }^{21,22}$

Según nuestro conocimiento, no se han publicado informes a largo plazo sobre el equipamiento completo de un paciente con hemicorporectomía.

Alcanzar una buena tolerancia de la prótesis aumenta notablemente la independencia del paciente y su aceptación..$^{23}$ Tal como ocurrió en nuestro paciente que, gracias a su equipamiento, logró una incorporación social casi completa y puntajes altos en las pruebas de resultados funcionales.

Por último, ante situaciones tan drásticas como la hemicorporectomía, la necesidad de un trabajo en equipo manteniendo al paciente motivado es fundamental para lograr buenos resultados.

\section{CONCLUSIONES}

La hemicorporectomía es una cirugía radical reservada para determinados casos terminales. El empleo de un equipo multidisciplinario es fundamental para alcanzar los objetivos. En pacientes motivados y con una rehabilitación adecuada, es posible lograr un equipamiento completo que le permita una incorporación social adecuada.

Conflicto de intereses: Los autores no declaran conflictos de intereses.

ORCID de G. L. Gallucci: https://orcid.org/0000-0002-0612-320X

\section{BIBLIOGRAFÍA}

1. Kredel FE. The role of pelvic evisceration in surgery (Discussion). Surgery 1951;30:76-94. PMID: 14845996

2. Kennedy CS, Miller EB, Mc Lean DS, Perlis MS, Dion RN, Horvitz VS. Lumbar ampution or hemicorporectomy for advanced malignancy of the lower half of the body. Surgery 1960;48:357-65. PMID: 14845996

3. Baker TC, Berkowitz T, Lord GB, Hankins HV. Hemicorporectomy. Br J Surg 1970;57(6):471-6. https://doi.org/10.1002/bjs.1800570617

4. Terz JJ, Schaffner MJ, Goodkin R, Beatty JD, Razor B, Weliky A, et al. Translumbar amputation. Cancer 1990;65:2668-75. https://doi.org/10.1002/1097-0142(19900615)65:12<2668::aid-cncr2820651212>3.0.co;2-i

5. Abrams J, Hulbert J, Thompson R, Cerra F, Delaney J. Hemicorporectomy for acute aortic occlusion: A case study. Am Surgeon 1992;58:509-12. PMID: 1642392

6. Friedmann LW, Marin EL, Park YS. Hemicorporectomy for functional rehabilitation. Arch Phys Med Rehabil 1981;62:83-6. PMID: 7235890

7. Aust JB, Page CP. Hemicorporectomy. J Surg Oncol 1985;30(4):226-30. https://doi.org/10.1002/jso.2930300408

8. Miller TR. Translumbar amputation (hemicorporectomy). Prog Clin Cancer 1982;8:227-36. PMID: 7156403

9. Miller TR, Mackenzie AR, Randall HT, Tigner SP. Hemicorporectomy. Surgery 1966;59(6):988-93. PMID: 5937972 
10. Richtr P, Hoch J, Svobodová K, Jech Z. Hemicorporectomy - the ultimate solution of terminal pelvic sepsis. Acta Chir Belg 2020;1-5. https://doi.org/10.1080/00015458.2020.1722930

11. Gómez F, Ramos Galvan R, Frenk S, Cravioto Muñoz J, Chávez R, Vázquez J. Mortality in second and third degree malnutrition. J Trop Ped (Lond) 1956;2:77-83. https://doi.org/ 10.1093/oxfordjournals.tropej.a057419

12. Ware JE Jr, Sherbourne CD. The MOS 36-item short-form health survey (SF-36) (I). Conceptual framework and item selection. Med Care 1992;30:473-83. PMID: 1593914

13. Mahoney FI, Barthel DW. Functional evaluation: the Barthel Index. Md State Med J 1965;13:61-5. PMID: 14258950

14. Grise MC, Gauthier-Gagnon C, Martineau GG. Prosthetic profile of people with lower extremity amputation: conception and design of a follow-up questionnaire. Arch Phys Med Rehabil 1993;74:862-70. https://doi.org/10.1016/0003-9993(93)90014-2

15. Shah S, Vanclay F, Cooper B. Improving the sensitivity of the Barthel Index for stroke rehabilitation. J Clin Epidemiol 1989;42:703-9. https://doi.org/10.1016/0895-4356(89)90065-6

16. Janis JE, Ahmad J, Lemmon JA, Barnett CC Jr, Morrill KC, Mc Clelland RN. A 25-year experience with hemicorporectomy for terminal pelvic osteomyelitis. Plast Reconstr Surg 2009;124(4):1165-76. https://doi.org/10.1097/PRS.0b013e3181b61169

17. Warr SP, Jaramillo PM, Franco ST, Valderrama-Molina CO, Franco AC. Hemicorporectomy as a life-saving strategy for severe pelvic ring crush injury: a case report. Eur J Orthop Surg Traumatol 2018;28(4):735-9. https://doi.org/10.1007/s00590-018-2140-z

18. Ferrara BE. Hemicorporectomy: a collective review. J Surg Oncol 1990;45(4):270-8. https://doi.org/10.1002/jso.2930450412

19. Barnett CC Jr, Ahmad J, Janis JE, Lemmon JA, Morrill KC, McClelland RN. Hemicorporectomy: back to front. Am J Surg 2008;196(6):1000-2. https://doi.org/10.1016/j.amjsurg.2008.08.009

20. Shields RK, Dudley-Javoroski S. Musculoskeletal deterioration and hemicorporectomy after spinal cord injury. Phys Ther 2003;83(3):263-75. PMID: 12620090

21. Smith J, Tuel SM, Meythaler JM, Cross LL, Schuch JZ. Prosthetic management of hemicorporectomy patients: new approaches. Arch Phys Med Rehabil 1992;73(5):493-7. https://doi.org/10.5555/uri:pii:000399939290

22. Simons BC, Lehman JF, Taylor N, De Lateur BJ. Prosthetic management of hemicorporectomy. Orthotics and Prosthetics 1968;22(2):63-8. Disponible en: http://www.oandplibrary.org/op/pdf/1968_02_063.pdf

23. Ludwigs, E, Kannenberg A, Wüstefeld D. Evaluation of the benefits of a new prosthetic hip joint system in activities of daily function in patients after hip disarticulation or hemipelvectomy. JPO Journal of Prosthetics and Orthotics 2013;25(3):118-26. https://doi.org/10.1097/JPO.0b013e31829afc1c 